\title{
miR-32-5p suppresses the proliferation and migration of pancreatic adenocarcinoma cells by targeting TLDC1
}

\author{
PENG YUAN $^{1 *}$, CHAOFENG TANG $^{1 *}$, BENDONG CHEN $^{1}$, PENG LEI $^{1}$, JIANJUN SONG $^{1}$, GUOJUN XIN $^{2}$, \\ ZUOZHENG WANG $^{1}$, YONGFENG HUI ${ }^{1}$, WEIJIE YAO ${ }^{1}$, GENWANG WANG ${ }^{1}$ and GUOZHONG ZHAO ${ }^{1}$ \\ ${ }^{1}$ Department of Hepatobiliary Surgery, General Hospital of Ningxia Medical University; ${ }^{2}$ Department of Hepatobiliary Surgery, \\ People's Hospital of Ningxia Hui Autonomous Region, Yinchuan, Ningxia Hui Autonomous Region 750004, P.R. China
}

Received November 30, 2020; Accepted July 12, 2021

DOI: $10.3892 / \mathrm{mmr} .2021 .12392$

\begin{abstract}
Pancreatic adenocarcinoma (PAAD) is one of the most fatal types of cancer in humans. However, the molecular mechanisms underlying the migration and invasion abilities of PAAD cells remain unclear. The aim of the present study was to explore the regulatory roles of microRNA (miR)-32-5p in PAAD cells. miR-32-5p mimic and inhibitor were used to transfect the human PAAD AsPC-1 cell line to determine the role of miR-32-5p in cell proliferation and metastasis. The starBase database predicted the binding of miR-32-5p to the target gene TBC/LysM-associated domain containing 1 (TLDC1). Further analyses were performed to assess miR-32-5p and TLDC1 expression levels in healthy and PAAD tissues, as well as the association between miR-32-5p or TLDC1 expression and the prognosis of patients with PAAD. The interaction between miR-32-5p and TLDC1 was verified using the dual-luciferase reporter assay. miR-32-5p and TLDC1 expression levels were detected by reverse transcription-quantitative PCR and western blotting, respectively. The Cell Counting Kit-8 assay was utilised to assess cell proliferation, whereas the wound-healing and Transwell assays were conducted to assess cell migration and invasion, respectively. miR-32-5p expression levels were markedly lower in PAAD tissue compared with those in healthy tissue, and were significantly lower in PAAD cell lines compared with those in the human pancreatic duct cell line HPDE6, which corresponded with poor prognosis. miR-32-5p significantly inhibited the proliferation of PAAD cells and markedly reduced migration and invasion
\end{abstract}

Correspondence to: Dr Guozhong Zhao, Department of Hepatobiliary Surgery, General Hospital of Ningxia Medical University, 804 Shengli South Street, Xingqing, Yinchuan, Ningxia Hui Autonomous Region 750004, P.R. China

E-mail: zhaogzh1220@163.com

${ }^{*}$ Contributed equally

Key words: microRNA-32-5p, TBC/LysM-associated domain containing 1, pancreatic adenocarcinoma, proliferation, invasion, migration compared with the negative controls. miR-32-5p was shown to target TLDC1, with miR-32-5p expression in PAAD being negatively correlated with TLDC1 expression. High TLDC1 expression levels were associated with a poorer prognosis compared with low TLDC1 expression levels. Co-transfection of miR-32-5p mimic and pcDNA/TLDC1 demonstrated that TLDC1 significantly reversed miR-32-5p-mediated inhibition of the proliferation, migration and invasion of PAAD cells. Overall, the present study demonstrated that miR-32-5p may serve as a tumor-suppressor gene by inhibiting the proliferation and migration and invasion of PAAD cells via the downregulation of TLDC1. Therefore, miR-32-5p may serve as a potential diagnostic or prognostic marker for PAAD.

\section{Introduction}

Pancreatic adenocarcinoma (PAAD) is a common gastrointestinal cancer associated with a high mortality rate, resulting in $>227,000$ deaths annually worldwide (1-3). The most common type of PAAD is pancreatic ductal adenocarcinoma, accounting for $80-90 \%$ of all pancreatic tumors (4). PAAD is among the most invasive types of carcinoma, and clinical diagnosis is often delayed due to a lack of known PAAD-specific symptoms (5). PAAD is therefore often diagnosed following metastasis and/or increases in cancer aggression, both of which greatly reduce the survival rate (6). The prognosis of PAAD is poor with a 1-year survival rate of $<10 \%$ (2). However, a definitive diagnosis of PAAD is not currently possible due to a lack of reliable tumor markers, which are urgently needed for initial detection and intervention (5). Currently, chemotherapy is the only available option for the treatment of advanced end-stage or metastatic PAAD (5).

MicroRNAs (miRNAs/miRs) are small, endogenous, non-coding RNAs that range in length from 19-25 nucleotides (7). miRNA can degrade or inhibit translation of mRNA by partial or total binding of the 3'-untranslated region (UTR) of the target molecule (7). miRNAs are key regulatory factors in various types of tumors, functioning as tumor oncogenes or tumor suppressors to regulate cell proliferation, differentiation, metastasis and apoptosis $(7,8)$. miR-32-5p, a member of the miR-32 family and located on chromosome band Xq26.2 (9), is involved in the regulation and development of numerous types of carcinoma. For example, Ye et al (10) reported that 
miR-32-5p inhibits the migration, invasion and proliferation of colorectal carcinoma cells; Wang et al (11) demonstrated that miR-32-5p represses the migration and invasion of clear cell renal cell carcinoma cells, and is positively correlated with a good prognosis; and Liu et al (12) indicated that miR-32-5p is downregulated in cervical carcinoma tissues and inhibits the proliferation, migration and invasion of cervical carcinoma cells.

TBC/LysM-associated domain-containing 1 (TLDC1) is an evolutionarily conserved protein 1 (13). Bioinformatics databases were analyzed to gain insight into the molecular functions of TLDC1, also known through genomic and proteomic studies as KIAA1609 (14), LOC57707 (15), or mEAK-7 (mammalian EAK-7 or MTOR associated protein, eak-7 homolog) (16). Independent reports reveal that TLDC1 mRNA is overexpressed in diseases, such as hepatocellular carcinoma (17) and lymph node-positive breast cancer (18). TLDC1 activates an alternative mTOR signaling pathway through S6K2 and 4E-BP1 to regulate cell proliferation and migration (13). miR-1911-3p targets mEAK-7 to suppress mTOR signaling in human lung cancer cells (19). it was hypothesized that miR-32-5p and TLDC1 may serve an important role in the development of PAAD.

Relatively few previous studies have investigated the role and underlying mechanisms of miR-32-5p in the pathogenesis of PAAD. For example, to the best of our knowledge, only one previous study has reported that growth arrest specific 5 could positively regulate PTEN-induced tumor suppressor pathway via miR-32-5p, thus inhibiting the metastasis of pancreatic cancer (20). Therefore, the aim of the present study was to investigate the relationship between miR-32-5p and TLDC1, and the underlying mechanisms in the onset and progression of PAAD.

\section{Materials and methods}

Cell culture. Human PAAD cell lines (BxPC-3, AsPC-1 and PANC-1) were obtained from American Type Culture Collection. PANC-1 and AsPC-1 cells were incubated in DMEM (Gibco; Thermo Fisher Scientific, Inc.) supplemented with $10 \%$ FBS, whereas BxPC-3 cells were incubated in RPMI medium (Gibco; Thermo Fisher Scientific, Inc.) supplemented with $10 \%$ FBS. The human pancreatic HPDE6 cell line was purchased from Shanghai Gefan Biotechnology Co., Ltd. and incubated in keratinocyte serum-free medium (Invitrogen; Thermo Fisher Scientific, Inc.) supplemented with human recombinant epidermal growth factor (Gibco; Thermo Fisher Scientific, Inc.) and bovine pituitary extract (Sigma-Aldrich; Merck $\mathrm{KGaA}$ ). All cells were maintained under $5 \% \mathrm{CO}_{2}$ at $37^{\circ} \mathrm{C}$.

Cell transfection. Human AsPC-1 cells were transfected with a negative control (NC) mimic, miR-32-5p mimic, NC inhibitor, miR-32-5p inhibitor (100 nM; all synthesized by Suzhou GenePharma Co., Ltd.), pcDNA control (empty vector) or pcDNA/TLDC1 (0.5 $\mu \mathrm{g}$; both obtained from GenScript) using Lipofectamine ${ }^{\circledR} 2000$ (Invitrogen; Thermo Fisher Scientific, Inc.) in accordance with the manufacturer's protocol. After incubation at $37^{\circ} \mathrm{C}$ for $48 \mathrm{~h}$, the cells were collected for subsequent assays. The sequences were as follows: NC mimic, 5'-UAUUGCACAUUACUAAGUUGCA-3', 5'-UAUUGCACA
UUACUAAGUUGCA-3'; miR-32-5p mimic (double-stranded RNA oligonucleotides without chemically modification), 5'-UAUUGCACAUUACUAAGUUGCA-3', 5'-CAACUUAGU AAUGUGCAAUAUU-3'; NC inhibitor, 5'-GUAUUCGAG UAAUCAACGAUAU-3'; and miR-32-5p inhibitor miR-96-5p inhibitor (single-stranded oligonucleotides chemically modified by 2'-Ome), 5'-UGCAACUUAGUAAUGUGCAAUA-3'. To verify successful transfection, reverse transcription-quantitative PCR (RT-qPCR) was performed to detect miR-32-5p expression levels at $12 \mathrm{~h}$ post-transfection, and western blotting was performed to detect protein expression levels $48 \mathrm{~h}$ post-transfection.

Bioinformatic analysis. miRNAs that regulate TLDC1 were predicted using starBase (http://starbase.sysu.edu.cn/) with TLDC1 as the key word.

Dual-luciferase reporter assay. The binding site between TLDC1 3'-UTR fragment and miR-32-5p were predicted using starBase (http://starbase.sysu.edu.cn/). To validate the microRNA-binding sequence of miR-32-5p, fragments containing wild-type or mutated TLDC1 3'-untranslated region (3'UTRs) of the predicted binding site were cloned into the pGL3 vector (Promega Corporation), which were constructed by Shanghai GeneChem Co., Ltd. AsPC-1 cells were seeded $\left(5 \times 10^{4}\right.$ cells/well) into 24 -well plates. Subsequently, cells were co-transfected with the reporter plasmid (TLDC1 3'UTR construct; $500 \mathrm{ng}$ ) and miR-32-5p mimic or NC mimic (900 ng) using Lipofectamine 2000 (Invitrogen; Thermo Fisher Scientific, Inc.) for $48 \mathrm{~h}$. Luciferase activity was detected using the Dual-Luciferase ${ }^{\circledR}$ Reporter Assay System (Promega Corporation) according to the manufacturer's protocol. Firefly luciferase activity was normalized to Renilla luciferase activity. The co-transfection systems were as follows: miR-32-5p mimic + TLDC1-WT; NC-mimic + TLDC1-WT; miR-32-5p mimic + TLDC1-MUT; and NC-mimic + TLDC1-MUT.

Cell Counting Kit-8 (CCK-8) assay. AsPC-1 cells $\left(4 \times 10^{3}\right.$ cells/well) were seeded into a 96 -well culture plate following incubation for $24,48,72$ and $96 \mathrm{~h}$ at $37^{\circ} \mathrm{C}$ with the CCK-8 reagent (ImmunoWay Biotechnology Company) according to the manufacturer's protocol, cell proliferation was measured at a wavelength of $450 \mathrm{~nm}$ using a microplate reader.

Wound-healing assay. Transfected human AsPC-1 cells were seeded $\left(2 \times 10^{4}\right.$ cells/well) into the wells of 6 -well plates and incubated in serum-free medium for $\sim 24 \mathrm{~h}$. At $100 \%$ confluence, a linear wound to the cell monolayer was made using the sterilized tip of a liquid pipette gun and the cells were cultured for $48 \mathrm{~h}$ at $37^{\circ} \mathrm{C}$. Subsequently, the media was discarded and the plates were washed three times in PBS. Plates were imaged and the serum-free medium was added prior to incubation for an additional $24 \mathrm{~h}$ at $37^{\circ} \mathrm{C}$. The plates were then imaged again after the 24-h incubation. Cells in randomly selected fields of view were counted using a light microscope (magnification, $\mathrm{x} 100$ ). The migratory ability of cells was indicated by gap closure. The assay was performed in triplicate.

Transwell assay. At $48 \mathrm{~h}$ post-transfection, AsPC-1 cells $\left(5 \times 10^{4}\right)$ were transferred to a Transwell chamber containing 
A hsa-miR-32-5p with 178 cancer and 4 normal samples in PAAD

Data source: starBase $\mathrm{v} 3.0$ project

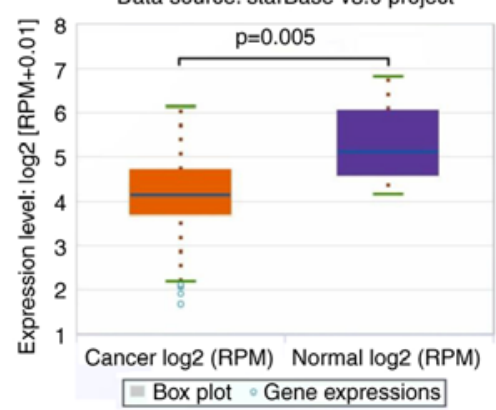

B 1.00 ty Overall survival for hsa-miR-32-5p in PAAD cancer Group

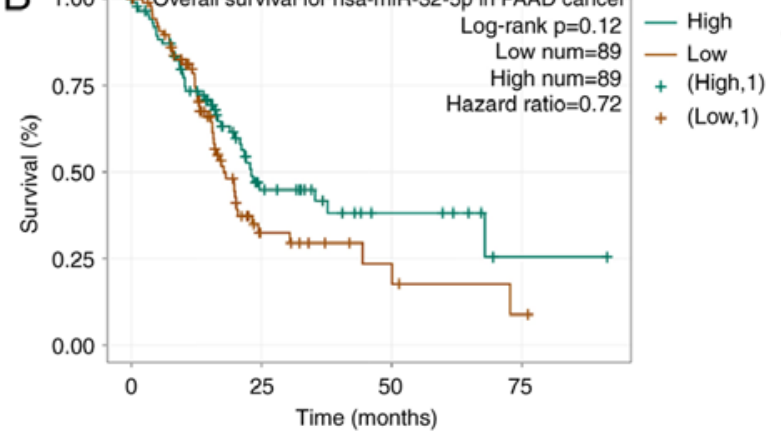

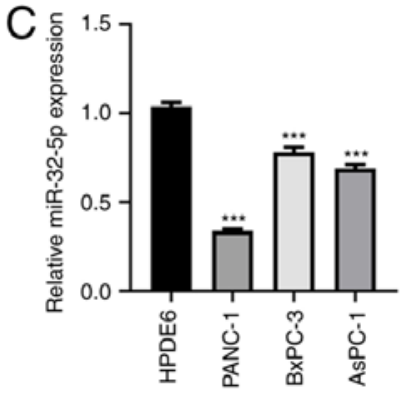

Figure 1. Expression levels of miR-32-5p in PAAD tissues and cells. (A) Expression of miR-32-5p in PAAD and healthy tissues was determined using the starBase database. (B) Relationship between miR-32-5p expression and the survival rate of patients with PAAD was determined using the starBase database. (C) Expression of miR-32-5p in HPDE6 cells and human PAAD cell lines (PANC-1, AsPC-1 and BxPC-3) detected by reverse transcription-quantitative PCR. ${ }^{* * *} \mathrm{P}<0.001$ vs. HPDE6. miR, microRNA; PAAD, pancreatic adenocarcinoma; RPM, reads per million.

Matrigel (BD Biosciences). Matrigel pre-coating was conducted for $30 \mathrm{~min}$ at $37^{\circ} \mathrm{C}$. Prior to conducting the assay, the lower and upper chambers were filled with pre-warmed media for hydration. Cells were then digested and resuspended in serum-free media. The upper chamber was loaded with cells suspended in serum-free media and the lower chamber with media supplemented with $10 \%$ FBS. The assay was conducted for $48 \mathrm{~h}$ at $37^{\circ} \mathrm{C}$. Following completion of the assay, the cells in the lower chamber were fixed for $30 \mathrm{~min}$ with $40 \%$ methanol at $25^{\circ} \mathrm{C}$ and then stained using $0.1 \%$ crystal violet for $20 \mathrm{~min}$ at $25^{\circ} \mathrm{C}$. Stained cells were visualized using an inverted light microscope (Olympus Corporation; magnification, x100).

Western blotting. Total protein was extracted from AsPC-1 cells using radioimmunoprecipitation assay buffer (Thermo Fisher Scientific, Inc.) containing a protease inhibitor cocktail (Roche Diagnostics) and quantified using a BCA kit (Beyotime Institute of Biotechnology,). Total protein (30 $\mu \mathrm{g} ; 25 \mu \mathrm{l} /$ well) was separated via $12 \%$ SDS-PAGE acrylamide gel. The separated proteins were then transferred onto a PVDF membrane, which was blocked with $5 \%$ skimmed milk in TBS containing $0.1 \%$ Tween- 20 for $1 \mathrm{~h}$ at $25^{\circ} \mathrm{C}$. The membranes were incubated overnight at $4^{\circ} \mathrm{C}$ with primary antibodies against TLDC1 (1:1,000; cat. no. AA426-456; 4A Biotech Co., Ltd.) and $\beta$-actin (1:2,000; Cell Signaling Technology Inc.). Following the primary incubation, membranes were washed three times with TBST $\left(0.05 \%\right.$ Tween-20) and incubated at $25^{\circ} \mathrm{C}$ for $2 \mathrm{~h}$ with secondary antibodies (horseradish peroxidase-conjugated goat anti-rabbit immunoglobulin G; 1:2,000; cat. no. ab6721; Abcam). The membranes were washed a further three times before visualization using ECL chemiluminescent detection system (Thermo Fisher Scientific, Inc.) according to the manufacturer's protocol. $\beta$-actin (1:2,000; cat. no. ab6276; Abcam) was used as the loading control. ImageJ version 1.46 software (National Institutes of Health) was used to semi-quantify protein expression.

$R T-q P C R$. Total RNA was extracted from AsPC-1 cells using TRIzol ${ }^{\circledR}$ reagent (Thermo Fisher Scientific, Inc.) according to the manufacturer's protocol. Total RNA was reverse transcribed into cDNA using the PrimeScript ${ }^{\mathrm{TM}}$ RT Reagent kit (Takara Biotechnology Co., Ltd.) according to the manufacturer's
Table I. Sequences of primers used for reverse transcription-quantitative PCR.

\begin{tabular}{ll}
\hline Gene & \multicolumn{1}{c}{ Sequence $\left(5^{\prime} \rightarrow 3^{\prime}\right)$} \\
\hline miR-32-5p & F: TAT TGC ACA TTA CTA AGC CTT \\
& R: GAA TAC CTC GGA CCC TGC \\
U6 & F: GCT TCG GCA GCA CAT ATA CTA AAA \\
& R: CGC TTC ACG AAT TTG CGT GTCAT \\
\hline
\end{tabular}

miR, microRNA; F, forward; R, reverse.

instructions. The expression of miR-32-5p was determined using a SYBR ${ }^{\circledR}$ Premix Ex Taq TM II (Tli RNaseH Plus) kit (Takara Bio, Inc.) according to the manufacturer's protocol. The following thermocycling conditions were used for $\mathrm{qPCR}$ : Pre-denaturation at $95^{\circ} \mathrm{C}$ for $30 \mathrm{sec}$; denaturation at $95^{\circ} \mathrm{C}$ for $10 \mathrm{sec}$; annealing and extension at $60^{\circ} \mathrm{C}$ for $30 \mathrm{sec}$ for 40 cycles. miR-32-5p expression levels were quantified using the $2^{-\Delta \Delta C q}$ method (21) and normalized to the internal reference gene, U6. The primers used for RT-qPCR are shown in Table 1.

Statistical analysis. All statistical analyses were performed using SPSS version 24.0 (IBM Corp.). Data are presented as the mean \pm SD. The unpaired Student's t-test was used to compare the means between two groups, whereas one-way ANOVA followed by Tukey's post-hoc test was used for multiple comparisons. Kaplan-Meier survival curves were analyzed using the log-rank test to determine statistical significance. Pearson's correlation coefficient test was used to determine whether there was a linear correlation between miR-32-5p and TLDC1 expression in PAAD. All experiments were repeated three times independently. $\mathrm{P}<0.05$ was considered to indicate a statistically significant difference.

\section{Results}

miR-32-5p is lowly expressed in PAAD tissues and cells. The starBase database was used to assess the expression pattern of miR-32-5p in PAAD and to identify the relationship between 

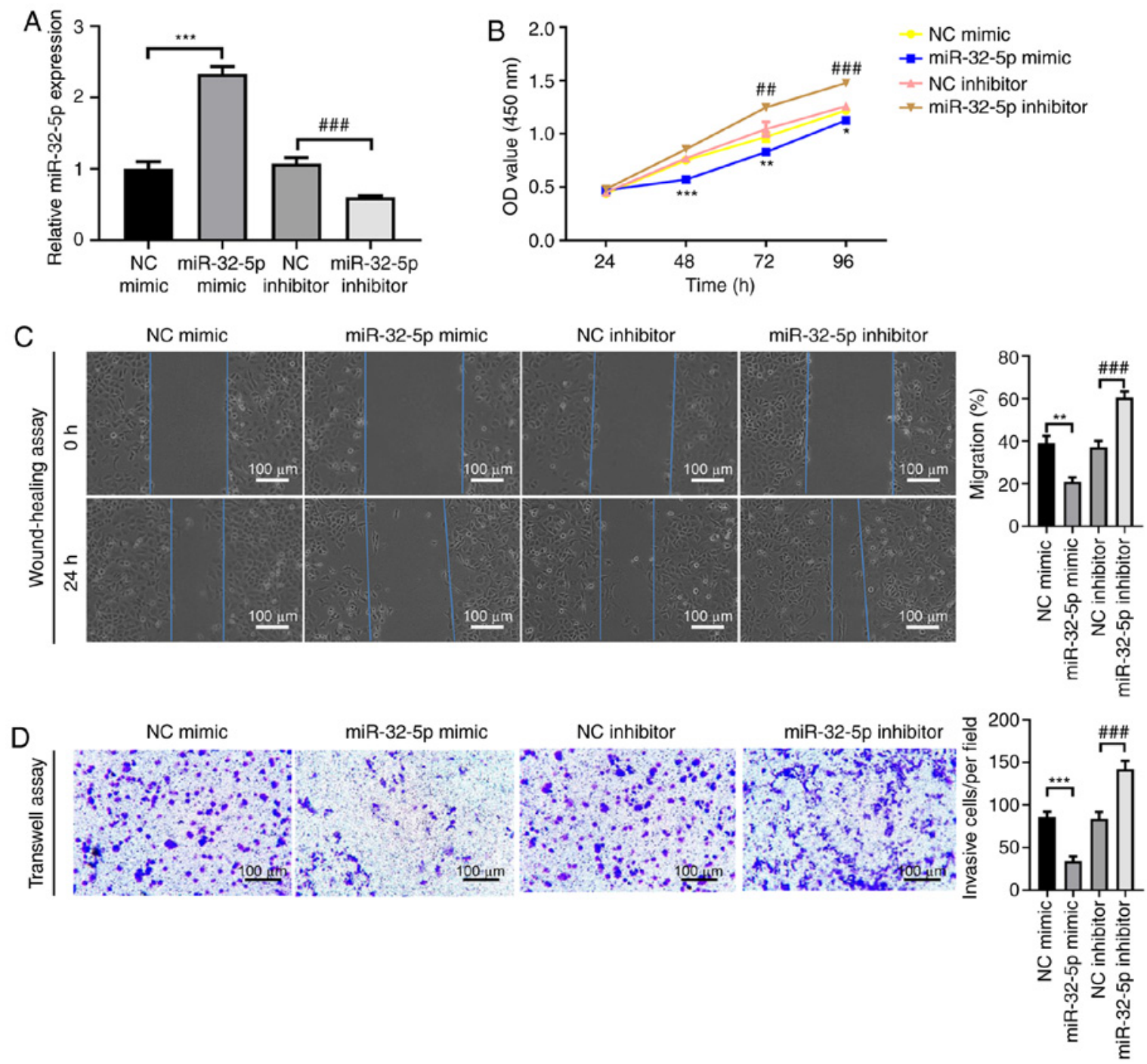

Figure 2. miR-32-5p inhibits the proliferation, migration and invasion of pancreatic adenocarcinoma cells. (A) miR-32-5p expression levels were detected at $72 \mathrm{~h}$ post-transfection using reverse transcription-quantitative PCR. (B) Effect of miR-32-5p expression on AsPC-1 cell proliferation was determined using the Cell Counting Kit-8 assay. (C) AsPC-1 cell migration was evaluated using the wound-healing assay. (D) AsPC-1 cell invasion was determined using the Transwell assay. ${ }^{*} \mathrm{P}<0.05,{ }^{* *} \mathrm{P}<0.01$ and ${ }^{* * * *} \mathrm{P}<0.001$ vs. $\mathrm{NC}$ mimic; ${ }^{\# \#} \mathrm{P}<0.01$ and ${ }^{\# \# \#} \mathrm{P}<0.001$ vs. NC inhibitor. miR, microRNA; NC, negative control; TLDC1, TBC/LysM-associated domain containing 1; OD, optical density.

miR-32-5p expression levels and PAAD prognosis. The results revealed that miR-32-5p expression was downregulated in the PAAD patient group compared with the healthy group (Fig. 1A; P<0.05). Low miR-32-5p expression was associated with a poor prognosis of patients with PAAD compared with high miR-32-5p expression (Fig. 1B; $\mathrm{P}=0.12$ ). Furthermore, miR-32-5p expression was measured in HPDE6 and PAAD cell lines (AsPC-1, PANC-1 and BxPC-3). The RT-qPCR results demonstrated that miR-32-5p expression levels were significantly reduced in the PAAD cells compared with those in HDPE6 cells (Fig. 1C; $\mathrm{P}<0.001$ ). These findings indicated that miR-32-5p might be involved in the development and progression of PAAD.

miR-32-5p inhibits the proliferation, migration and invasion of PAAD cells. To assess the effect of miR-32-5p overexpression and knockdown, the AsPC-1 cell line was selected due to moderate miR-32-5p expression levels. To determine whether miR-32-5p was associated with the proliferation, migration and invasion of PAAD cells, functional studies of AsPC-1 cells were performed. The results demonstrated that miR-32-5p mimic caused a significant increase in miR-32-5p mRNA expression compared with NC mimic, whereas miR-32-5p inhibitor significantly reduced miR-31-5p expression compared with NC inhibitor. This indicated the successful establishment of miR-32-5p overexpression and knockdown in AsPC-1 cells (Fig. 2A; both $\mathrm{P}<0.001$ ). The CCK-8 assay results revealed that miR-32-5p mimic significantly impaired the proliferation of AsPC-1 compared with $\mathrm{NC}$ mimic (Fig. 2B; $\mathrm{P}<0.001$ at $48 \mathrm{~h} ; \mathrm{P}<0.01$ at $72 \mathrm{~h} ; \mathrm{P}<0.05$ at $96 \mathrm{~h}$ ). The metastatic potential of AsPC-1 cells was measured using wound-healing and Transwell assays. Cell migration was significantly impaired in miR-32-5p mimic-transfected cells compared with NC mimic-transfected cells (Fig. 2C; $\mathrm{P}<0.01)$. miR-32-5p knockdown significantly increased cell proliferation (Fig. 2B; $\mathrm{P}<0.01$ at $72 \mathrm{~h} ; \mathrm{P}<0.001$ at $96 \mathrm{~h}$ ), and significantly increased cell migration (Fig. $2 \mathrm{C} ; \mathrm{P}<0.001$ ) and invasion compared with $\mathrm{NC}$ inhibitor (Fig. 2D; $\mathrm{P}<0.001$ ). 
A hsa-miR-32-5p 3' ACGUUGAAUCAUUACACGUUAU 5' || || ||||||

TLDC1 $5^{\prime}$ UguUUUUUAUAaAaAugCAAUU $\quad 3$ '
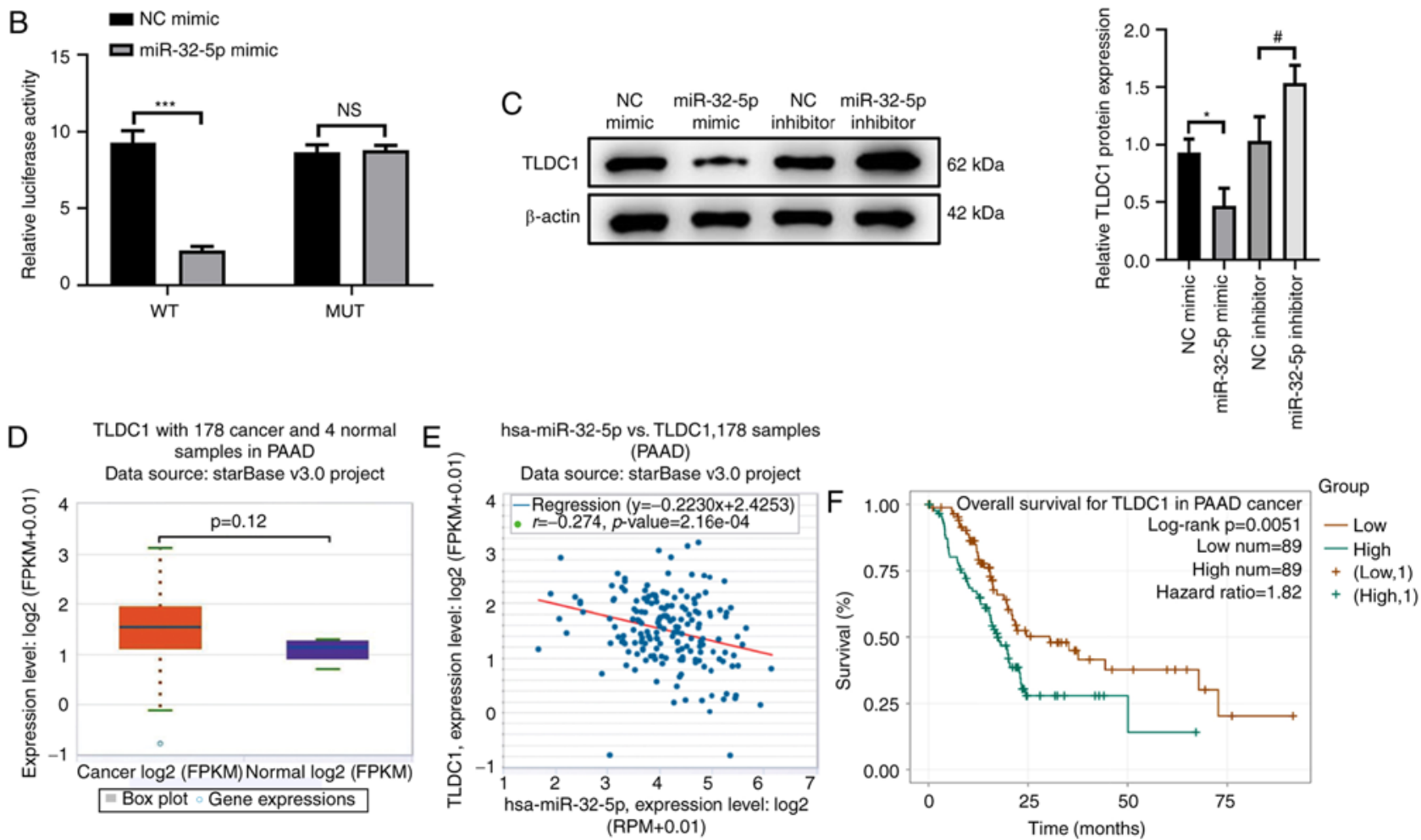

Figure 3. TLDC1 is a direct target gene of miR-32-5p in PAAD. (A) Binding sites between miR-32-5p and TLDC1 were predicted using the starBase database. (B) Luciferase activity of the reporter plasmid containing either WT or MUT TLDC1 3' untranslated region in AsPC-1 cells following co-transfection with miR-32-5p mimic or NC mimic. (C) TLDC1 protein expression levels determined using western blotting. (D) mRNA expression levels of TLDC1 in PAAD tissues and healthy tissues determined using the starBase database. (E) Relationship between miR-32-5p and TLDC1 expression levels in PAAD samples determined using the starBase database. (F) Relationship between TLDC1 expression levels and the overall survival rate of patients with PAAD determined using the starBase database. ${ }^{*} \mathrm{P}<0.05$ and ${ }^{* * * *} \mathrm{P}<0.001$ vs. NC mimic; ${ }^{*} \mathrm{P}<0.05$ vs. NC inhibitor. TLDC1, TBC/LysM-associated domain containing 1 ; miR, microRNA; PAAD, pancreatic adenocarcinoma; WT, wild-type; MUT, mutant; NS, not significant; NC, negative control; FPKM, fragments per kilobase million; RPM, reads per million.

Collectively, these findings demonstrated that miR-32-5p inhibited the proliferation and metastatic potential of PAAD cells.

$T L D C 1$ is a target of $m i R-32-5 p$. To elucidate the downstream molecular mechanisms of miR-32-5p in the proliferation, migration and invasion of PAAD cells, the starBase database was searched to identify the target sequence of miR-32-5p. The results demonstrated that miR-32-5p targeted the 3'-UTR of TLDC1 (Fig. 3A). The dual-luciferase reporter assay was performed to confirm the interaction between miR-32-5p and TLDC1. The results demonstrated that luciferase activity was significantly reduced in cells co-transfected with TLDC1-WT and miR-32-5p mimic compared with those co-transfected with NC mimic $(\mathrm{P}<0.001)$. No significant difference in luciferase activity was observed in the MUT TLDC1 co-transfection system, confirming that miR-32-5p targeted TLDC1 (Fig. 3B; P $>0.05$ ). Moreover, TLDC1 protein expression levels were significantly reduced in cells transfected with miR-32-5p mimic compared with the NC mimic group, whereas miR-32-5p inhibitor significantly increased the protein expression levels of TLDC1 compared with the NC inhibitor group (Fig. 3C; both $\mathrm{P}<0.05)$. These results demonstrated that miR-32-5p could both target and regulate TLDC1 in PAAD cells, which was further confirmed by bioinformatics analyses. The starBase database demonstrated that TLDC1 expression was increased in PAAD cells compared with the NC group (Fig. 3D; $\mathrm{P}=0.12$ ). Furthermore, TLDC1 expression levels were negatively correlated with miR-32-5p in PAAD tissues (Fig. 3E; P<0.05). High TLDC1 expression levels were significantly associated with a better prognosis of patients with PAAD compared with lower TLDC expression levels (Fig. 3F; $\mathrm{P}<0.05$ ). Overall, these results indicated that TLDC1 may serve a regulatory role in the proliferation, migration and invasion of PAAD cells via miR-32-5p.

$T L D C 1$ reverses the suppressive effect of $m i R-32-5 p$ on the proliferation, migration and invasion of PAAD cells. To explore the effect of TLDC1 on the proliferation, migration and invasion of PAAD cells mediated by miR-32-5p, simultaneous overexpression of TLDC1 and miR-32-5p was induced. The results demonstrated that TLDC1 protein expression levels were significantly increased in cells transfected with NC + pcDNA/TLDC1 compared with those transfected with pcDNA/control, indicating that TLDC1 was successfully overexpressed (Fig. 4A; all $\mathrm{P}<0.001$ ). Transfection with miR-32-5p mimic significantly reduced TLDC1 protein expression levels compared with the 

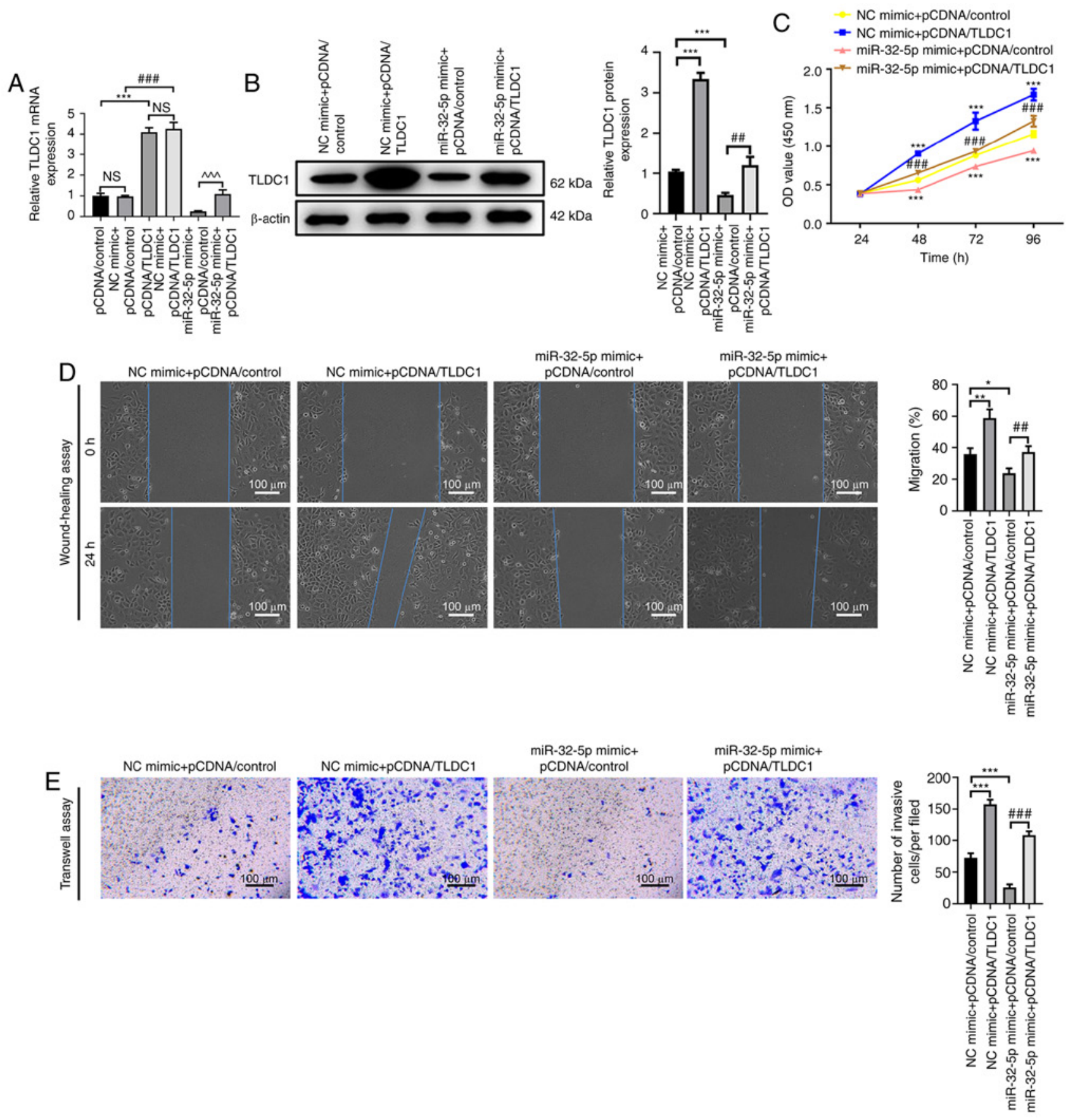

Figure 4. TLDC1 overexpression rescues miR-32-5p-mediated inhibition of the proliferation, migration and invasion of pancreatic adenocarcinoma cells. TLDC1 (A) mRNA and (B) protein expression levels were determined by reverse transcription-quantitative PCR and western blotting, respectively. ${ }^{* * *} \mathrm{P}<0.01 \mathrm{vs}$. pcDNA/ control; ${ }^{\# \# \prime} \mathrm{P}<0.001$ vs. NC mimic + pcDNA/control; ${ }^{\wedge \wedge} \mathrm{P}<0.001$ vs. miR-32-5p mimic + pcDNA/control. Effect of miR-32-5p and TLDC1 overexpression on the (C) proliferation (D) migration and (E) invasion of AsPC-1 cells determined using Cell Counting Kit-8, wound-healing and Transwell assays, respectively. "P $<0.05$, ${ }^{* *} \mathrm{P}<0.01$ and ${ }^{* * *} \mathrm{P}<0.01$ vs. NC mimic + pcDNA/control; "\# $\mathrm{P}<0.05$ and ${ }^{\# \# \#} \mathrm{P}<0.001$ vs. miR-32-5p mimic. TLDC1, TBC/LysM-associated domain containing $1 ;$ miR, microRNA; OD, optical density.

$\mathrm{NC}+$ pcDNA/control group. However, co-transfection with miR-32-5p and TLDC1 significantly reversed the decrease in TLDC1 protein expression levels induced by miR-32-5p compared with miR-32-5p + pcDNA/control (Fig. 4B; P<0.01). Compared with the NC + pcDNA/control group, miR-32-5p overexpression was also shown to significantly impair the proliferation of AsPC-1 cells, whereas TLDC1 overexpression significantly reversed miR-32-5p mimic-mediated effects (Fig. 4C; both $\mathrm{P}<0.001$ ). The wound-healing assay results showed that cell migration was significantly decreased in the
miR-32-5p mimic + pCDNA/control group compared with that in the NC mimic + pCDNA/control group $(\mathrm{P}<0.05)$, and TLDC1 overexpression significantly reversed miR-32-5p mimic-mediated inhibition of migration (Fig. 4D; $\mathrm{P}<0.01$ ). Furthermore, the Transwell assay results indicated miR-32-5p overexpression significantly decreased the number of invasive cells compared with the NC mimic + pCDNA/control group $(\mathrm{P}<0.001)$, and TLDC1 overexpression partially restored this decrease (Fig. 4E; $\mathrm{P}<0.001)$. In summary, these data suggested that TLDC1 was a target of miR-32-5p and reversed 
miR-32-5p-mediated inhibition of the proliferation, migration and invasion of PAAD cells.

\section{Discussion}

PAAD is characterized by poor prognosis and resistance to therapy, which explains the relatively high mortality rate compared with other types of cancer (22-24). At present, the treatment options for PAAD are very limited, with surgical resection of the tumor remaining the most effective (25-27). However, for the majority of patients with PAAD, surgery is not a viable option due to a high chance of a relapse occurring $(23,28,29)$. Therapeutic prospects for PAAD remain poor even with improved treatment strategies because of the high risk of metastasis $(23,30,31)$. Due to the complicated configuration of the adjoining anatomy of the pancreas, whereby the pancreas is in contact with numerous neighboring structures, pancreatic cancer cells tend to also aggressively attack the adjacent tissues $(24,25,30,32)$. Therefore, it is essential to identify the mechanisms underlying the proliferation and metastasis of PAAD.

Previous studies have determined that multiple miRNAs can influence the occurrence and progression of PAAD by modulating the expression of various target genes. Zhang et al (33) reported that miR-135b-5p contributed to the epithelial-mesenchymal transition, migration and invasion of PAAD cells by targeting the nuclear receptor protein, nuclear receptor subfamily 3 group C member 2. Furthermore, Daniel et al (34) revealed that miR-32-5p is downregulated in prostate cancer tissues, whereas Wang et al (35) reported that miR-32-5p is lowly expressed in triple-negative breast cancer. Collectively, these previous reports suggest that miR-32-5p regulates the development of various types of cancer to a certain degree. Hence, the aim of the present study was to investigate the role, and identify the target genes and physiological functions of miR-32-5p in PAAD. The results demonstrated that miR-32-5p expression levels were markedly reduced in PAAD tissues and cell lines compared with healthy tissues and healthy human pancreatic cells. Moreover, elevated miR-32-5p expression levels were associated with a better prognosis of patients with PAAD compared with those with lower miR-32-5p expression levels, which indicated that miR-32-5p may display a tumor-suppressive effect in the onset and progression of PAAD.

Based on the differential expression of miR-32-5p in PAAD, it was speculated that miR-32-5p may be associated with the proliferation, migration and invasion of PAAD. The present study demonstrated that overexpression of miR-32-5p significantly suppressed the proliferation and markedly decreased the migration and invasion of PAAD cells. Collectively, these results suggested that miR-32-5p had an antitumor effect in PAAD cells, serving an oncogenic role in the progression of PAAD. Therefore, miR-32-5p may have the potential to function as a diagnostic biomarker and therapeutic target for PAAD.

Various miRNAs have been shown to serve roles in tumor development and progression by mediating cell proliferation and metastasis. miR-337-3p has been reported to regulate the proliferation, invasion, migration and apoptosis of cervical cancer cells by targeting Ras-related protein Rap1A expression (36), whereas miR-124 was found to regulate metastasis and the epithelial-mesenchymal transition in triple-negative breast cancer by targeting zinc finger E-box-binding homeobox 2 protein expression (37). Furthermore, miR-335 was demonstrated to suppress the proliferation of lung cancer cells by targeting transformer- 2 protein homolog $\beta$ expression (38). Further identification of miR-32-5p target genes will help to further elucidate the role of miR-32-5p in the pathogenesis of PAAD. miRNAs generally bind to the 3'-UTR of target mRNAs (39). In the present study, the starBase database was searched to predict the direct target gene of miR-32-5p in PAAD, which identified TLDC1. Furthermore, these results demonstrated that TLDC1 expression was relatively higher in PAAD tissue compared with that in healthy tissue and negatively correlated with miR-32-5p expression in patients with PAAD. Patients with lower TLDC1 protein expression levels exhibited a better prognosis compared with those displaying higher TLDC protein expression levels. The present study therefore indicated that TLDC1 may serve a negative regulatory function in the development of PAAD induced by miR-32-5p.

miR-32-5p serves a key biological role in the tumorigenesis of various types of cancer by regulating multiple target genes. In cervical cancer, homeobox B8 is reported to reverse the proliferation, invasion and migration of cells inhibited by miR-32-5p (12). In colorectal cancer, the transducer of ERRB2-1 protein was found to reverse the inhibitory effect of miR-32-5p on cell sensitization and the promoting effect of miR-32-5p on cell migration and invasion (7). In ovarian cancer, SMG1 nonsense mediated mRNA decay-associated PI3K-related kinase was shown to reduce the promoting effect of miR-32-5p on cell proliferation and motility (9). The present study demonstrated that miR-32-5p suppressed the proliferation, migration and invasion of PAAD cells, which was rescued by TLDC1 overexpression.

The present study revealed that miR-32-5p was lowly expressed in PAAD. miR-32-5p was shown to negatively target TLDC1, inhibiting the proliferation, migration, and invasion of PAAD cells. As a tumor suppressor gene, miR-32-5p may serve as a promising biomarker for the diagnosis and prognosis of PAAD. However the present study had a number of limitations. For example, the results were not verified using animal models or other PAAD cells. Moreover, the upstream regulation of miR-32-5p and the pathways involved in TLDC1 requires further investigation.

\section{Acknowledgements}

Not applicable.

\section{Funding}

No funding was received.

\section{Availability of data and materials}

The datasets used and/or analyzed during the current study are available from the corresponding author on reasonable request.

\section{Authors' contributions}

PY and CT designed the study and wrote the manuscript with contributions from all authors. All authors participated in 
performing the experiments. BC, PL, JS, GX, ZW, and GZ were responsible for data acquisition and the interpretation of data. YH, WY and GW were responsible for statistical analysis, the literature search and revising the article critically for important intellectual content. All authors read and approved the final manuscript. PY, CT and GZ confirm the authenticity of all the raw data.

\section{Ethics approval and consent to participate}

Not applicable.

\section{Patient consent for publication}

Not applicable.

\section{Competing interests}

The authors declare that they have no competing interests.

\section{References}

1. Xu B, Liu J, Xiang X, Liu S, Zhong P, Xie F, Mou T and Lai L: Expression of miRNA-143 in pancreatic cancer and its clinical significance. Cancer Biother Radiopharm 33: 373-379, 2018.

2. Yu Z, Zhao S, Wang L, Wang J and Zhou J: MiRNA-339-5p plays an important role in invasion and migration of pancreatic cancer cells. Med Sci Monit 25: 7509-7517, 2019.

3. Du W, Lei C, Wang Y, Ding Y and Tian P: LINC01232 sponges multiple miRNAs and its clinical significance in pancreatic adenocarcinoma diagnosis and prognosis. Technol Cancer Res Treat 20: 1533033820988525,2021

4. Wang C, Huang Y,Zhang J and Fang Y: MiRNA-339-5p suppresses the malignant development of gastric cancer via targeting ALKBH1. Exp Mol Pathol 115: 104449, 2020.

5. Hessmann E, Buchholz SM, Demir IE, Singh SK, Gress TM, Ellenrieder V and Neesse A: Microenvironmental determinants of pancreatic cancer. Physiol Rev 100: 1707-1751, 2020.

6. Leinwand $\mathrm{J}$ and Miller G: Regulation and modulation of antitumor immunity in pancreatic cancer. Nat Immunol 21: 1152-1159, 2020.

7. Liang $\mathrm{H}$, Tang $\mathrm{Y}$, Zhang $\mathrm{H}$ and Zhang C: MiR-32-5p regulates radiosensitization, migration and invasion of colorectal cancer cells by targeting TOB1 gene. Onco Targets Ther 12: 9651-9661, 2019.

8. Du B, Zhang P, Tan Z and Xu J: MiR-1202 suppresses hepatocellular carcinoma cells migration and invasion by targeting cyclin dependent kinase 14. Biomed Pharmacother 96: 1246-1252, 2017.

9. Zeng S, Liu S, Feng J, Gao J and Xue F: MicroRNA-32 promotes ovarian cancer cell proliferation and motility by targeting SMG1. Oncol Lett 20: 733-741, 2020.

10. Ye T, Zhang N, Wu W, Yang B, Wang J, Huang W and Tang D: SNHG14 promotes the tumorigenesis and metastasis of colorectal cancer through miR-32-5p/SKIL axis. In Vitro Cell Dev Biol Anim 55: 812-820, 2019.

11. Wang M, Sun Y, Xu J, Lu J, Wang K, Yang DR, Yang G, Li G and Chang C: Preclinical studies using miR-32-5p to suppress clear cell renal cell carcinoma metastasis via altering the miR-32-5p/TR4/HGF/Met signaling. Int J Cancer 143: 100-112, 2018.

12. Liu YJ, Zhou HG, Chen LH, Qu DC, Wang CJ, Xia ZY and Zheng JH: MiR-32-5p regulates the proliferation and metastasis of cervical cancer cells by targeting HOXB8. Eur Rev Med Pharmacol Sci 23: 87-95, 2019.

13. Nguyen JT, Ray C, Fox AL, Mendonça DB, Kim JK and Krebsbach PH: Mammalian EAK-7 activates alternative mTOR signaling to regulate cell proliferation and migration. Sci Adv 4: eaao5838, 2018.

14. Nagase T, Kikuno R, Nakayama M, Hirosawa M and Ohara O: Prediction of the coding sequences of unidentified human genes. XVIII. The complete sequences of 100 new cDNA clones from brain which code for large proteins in vitro. DNA Res 7: 273-281, 2000.
15. Schröder B, Wrocklage C, Pan C, Jäger R, Kösters B, Schäfer H, Elsässer HP, Mann M and Hasilik A: Integral and associated lysosomal membrane proteins. Traffic 8: 1676-1686, 2007.

16. Nguyen JT, Haidar FS, Fox AL, Ray C, Mendonça DB, Kim JK and Krebsbach PH: mEAK-7 forms an alternative mTOR complex with DNA-PKcs in human cancer. iScience 17: 190-207, 2019.

17. Riou P, Saffroy R, Comoy J, Gross-Goupil M, Thiéry JP, Emile JF, Azoulay D, Piatier-Tonneau D, Lemoine A and Debuire B: Investigation in liver tissues and cell lines of the transcription of 13 genes mapping to the $16 \mathrm{q} 24$ region that are frequently deleted in hepatocellular carcinoma. Clin Cancer Res 8: 3178-3186, 2002.

18. Ellsworth RE, Field LA, Love B, Kane JL, Hooke JA and Shriver CD: Differential gene expression in primary breast tumors associated with lymph node metastasis. Int J Breast Cancer 2011: $142763,2011$.

19. Mendonça DB, Nguyen JT, Haidar F, Fox AL, Ray C, Amatullah H,LiuF,KimJK and Krebsbach PH:MicroRNA-1911-3p targets mEAK-7 to suppress mTOR signaling in human lung cancer cells. Heliyon 6: e05734, 2020.

20. Gao ZQ, Wang JF, Chen DH, Ma XS, Wu Y, Tang Z and Dang XW: Long non-coding RNA GAS5 suppresses pancreatic cancer metastasis through modulating miR-32-5p/PTEN axis. Cell Biosci 7: 66, 2017.

21. Livak KJ and Schmittgen TD: Analysis of relative gene expression data using real-time quantitative PCR and the 2(-Delta Delta C(T)) method. Methods 25: 402-408, 2001.

22. Siegel RL, Miller KD and Jemal A: Cancer statistics, 2015. CA Cancer J Clin 65: 5-29, 2015.

23. Cao J, Yang J, Ramachandran V, Arumugam T, Deng DF, Li ZS, Xu LM and Logsdon CD: TM4SF1 regulates pancreatic cancer migration and invasion in vitro and in vivo. Cell Physiol Biochem 39: 740-750, 2016.

24. Xu Q, Zong L, Chen X, Jiang Z, Nan L, Li J, Duan W, Lei J, Zhang L, Ma J, et al: Resveratrol in the treatment of pancreatic cancer. Ann N Y Acad Sci 1348: 10-19, 2015.

25. Cao L, Chen X, Xiao X, Ma Q and Li W: Resveratrol inhibits hyperglycemia-driven ROS-induced invasion and migration of pancreatic cancer cells via suppression of the ERK and p38 MAPK signaling pathways. Int J Oncol 49: 735-743, 2016.

26. Koay EJ, Amer AM, Baio FE, Ondari AO and Fleming JB: Toward stratification of patients with pancreatic cancer: Past lessons from traditional approaches and future applications with physical biomarkers. Cancer Lett 381: 237-243, 2016.

27. Sabater L, Muñoz E, Roselló S, Dorcaratto D, Garcés-Albir M, Huerta M, Roda D, Gómez-Mateo MC, Ferrández-Izquierdo A, Darder A and Cervantes A: Borderline resectable pancreatic cancer. Challenges and controversies. Cancer Treat Rev 68: 124-135, 2018.

28. Hartwig W, Werner J, Jäger D, Debus J and Büchler MW: Improvement of surgical results for pancreatic cancer. Lancet Oncol 14: e476-e485, 2013.

29. Kamisawa T, Wood LD, Itoi $\mathrm{T}$ and Takaori K: Pancreatic cancer. Lancet 388: 73-85, 2016.

30. Qian B, Wei L, Yang Z, He Q, Chen H, Wang A, Yang D, Li Q, Li J, Zheng S and Fu W: Hic-5 in pancreatic stellate cells affects proliferation, apoptosis, migration, invasion of pancreatic cancer cells and postoperative survival time of pancreatic cancer. Biomed Pharmacother 121: 109355, 2020.

31. Middleton G, Palmer DH, Greenhalf W, Ghaneh P, Jackson R, Cox T, Evans A, Shaw VE, Wadsley J, Valle JW, et al: Vandetanib plus gemcitabine versus placebo plus gemcitabine in locally advanced or metastatic pancreatic carcinoma (ViP): A prospective, randomised, double-blind, multicentre phase 2 trial. Lancet Oncol 18: 486-499, 2017.

32. Hartwig W, Vollmer CM, Fingerhut A, Yeo CJ, Neoptolemos JP, Adham M, Andrén-Sandberg A, Asbun HJ, Bassi C, Bockhorn M, et al: Extended pancreatectomy in pancreatic ductal adenocarcinoma: Definition and consensus of the International Study Group for Pancreatic Surgery (ISGPS). Surgery 156: 1-14, 2014.

33. Zhang Z, Che X, Yang N, Bai Z, Wu Y, Zhao L and Pei H: miR$135 \mathrm{~b}-5 \mathrm{p}$ Promotes migration, invasion and EMT of pancreatic cancer cells by targeting NR3C2. Biomed Pharmacother 96: 13411348, 2017.

34. Daniel R, Wu Q, Williams V, Clark G, Guruli G and Zehner Z: A Panel of MicroRNAs as diagnostic biomarkers for the identification of prostate cancer. Int J Mol Sci 18: 1281, 2017.

35. Wang R, Huang Z, Qian C, Wang M, Zheng Y, Jiang R and Yu C: LncRNA WEE2-AS1 promotes proliferation and inhibits apoptosis in triple negative breast cancer cells via regulating miR-32-5p/TOB1 axis. Biochem Biophys Res Commun 526: 1005-1012, 2020. 
36. Cao XM: Role of miR-337-3p and its target Rap1A in modulating proliferation, invasion, migration and apoptosis of cervical cancer cells. Cancer Biomark 24: 257-267, 2019.

37. Ji H, Sang M, Liu F, Ai N and Geng C: MiR-124 regulates EMT based on ZEB2 target to inhibit invasion and metastasis in triple-negative breast cancer. Pathol Res Pract 215: 697-704, 2019.

38. Liu J, Bian T, Feng J, Qian L, Zhang J, Jiang D, Zhang Q, Li X, Liu Y and Shi J: MiR-335 inhibited cell proliferation of lung cancer cells by target Tra2 $\beta$. Cancer Sci 109: 289-296, 2018.
39. Cannell IG, Kong YW and Bushell M: How do microRNAs regulate gene expression? Biochem Soc Trans 36 (Pt 6): 1224-1231, 2008 .

c) (i) $\odot$ This work is licensed under a Creative Commons

cc) Attribution-NonCommercial-NoDerivatives 4.0 International (CC BY-NC-ND 4.0) License. 